\title{
A Simple Numerical Method For Van der Pol- Duffing Oscillator Equation
}

\author{
Chengli Zhang \\ Faculty of Metallurgical and Energy Engineering \\ Kunming University of Science and Technology \\ Kunming, China \\ e-mail: Zhangchengli1118@163.com
}

\author{
Yun Zeng \\ Department of Engineering Mechanics \\ Kunming University of Science and Technology \\ Kunming, China \\ e-mail: zengyun001@163.com
}

\begin{abstract}
The famous Van der Pol-Duffing oscillator is a typical nonlinear oscillator, its accurate analytical solution can not yet directly be got now. The segmenting recursion method was put forward in this paper, the oscillator equation was reasonably linearized within small segment, then the approximate analytical solutions within small segment were got, and the recursion formulas were obtained, finally all numerical solutions were got by the recursion formulas. The numerical solutions of several Van der PolDuffing oscillator equation were got by means of the segmenting recursion method, and the numerical solutions were compared with Runge-Kutta method or Gear linear multistep method. The comparative results show that the segmenting recursion method is very effective to solve Van der Pol-Duffing oscillator equation, not only method is simple, programming is easy, butresult is accurate, this method is a universal new method to solve similar oscillator.
\end{abstract}

Keywords- Van der Pol-Duffing; nonlinear; linearization; segmenting method; recursion method

\section{INTRODUCTION}

The famous Van der Pol-Duffing oscillator is a typical nonlinear oscillator, which has rich dynamical behaviors, widely exists in electronics, circuit, mechanics, finance and other models, has important research value and wide application background, is often used to verify the effectiveness and accuracy of new method in [1] - [5].

The accurate analytical solution of Van der PolDuffing oscillator equation can not yet be obtained now in [6] - [8], thus judgment method and asymptotic method were widely applied in [9] - [12]. Judgment method is mainly used for nonlinear control system to avoid directly solving equations in [13]. Asymptotic method is mainly used for mathematics and mechanics fields, such as phase plane method, describing function method, approximate analytical method and numerical method, and so on in [14]. Approximate analytical method mainly includes linearization method, perturbation method, homotopy analysis method, variational iteration method, etc. in [15]. Numerical method is applied widely, the traditional method includes Euler method, Runge-Kutta method and linear multistep method, etc. the newer method includes differential geometry theory, geometric integration theory, differential algebraic method, etc. in [16].

Linearization method mainly includes Taylor series method, small signal analysis method, least square method, neglecting higher order small quantity method, segmenting method, and so on in [17]. Segmenting method divides working area into many small segments, and the curves within small segment are approximately replaced by straight lines in [18], then the approximate analytical solutions within small segments are obtained. In principle, segmenting method is suitable for all nonlinear system.

The segmenting recursion method put forward in this paper is a simple new method combined segmenting method with recursion method. First, the equation within small segment is linearized by segmenting, and then the recursion formulas were obtained by solving the approximate analytical solution within small segment, finally, all numerical solutions are got based on the recursion formulas. The numerical solutions of several Van der Pol-Duffing oscillator were got by means of the segmenting recursion method, and the numerical solutions were compared with Runge-Kutta method or Gear linear multistep method to verify the effectiveness solving Van der Pol-Duffing oscillator, then step length selection is analyzed.

\section{SEGMENTING RECURSION METHOD}

Van der Pol-Duffing forced oscillator equation is as follows:

$$
\left\{\begin{array}{l}
\frac{d^{2} y}{d t^{2}}+\left(\varepsilon+\mu y^{2}\right) \frac{d y}{d t}+\alpha y+\beta y^{3}=b \cos (\omega t) \\
y(0)=y_{0}, \frac{d y}{d t}(0)=v_{0}
\end{array}\right.
$$

where $\varepsilon$ is linear damping coefficient, $\mu$ is nonlinear damping coefficient, $\alpha$ is linear item coefficient, $\beta$ is nonlinear stiffness coefficient, $b$ is excitation amplitude, $\omega$ is excitation frequency, $y_{0}$ is the initial value of $y$ at $t=0$, $v_{0}$ is the initial value of $d y / d t$ at $t=0$.

When $b=0,(1)$ degrades into self-excitation oscillator equation, when $\beta=0$, (1) degrades into Van der Pol oscillator equation; When $\mu=0$, (1) degrades into Duffing oscillator equation.

Equation (1) is organized into the following form:

$$
\frac{d^{2} y}{d t^{2}}+A \frac{d y}{d t}=B, \quad y(0)=y_{0}, \quad \frac{d y}{d t}(0)=v_{0}
$$

where $A=\varepsilon+\mu y^{2}, B=b \cos (\omega t)-\alpha y-\beta y^{3}$. 
The specific steps solving (2) by segmenting recursive method (or SRM for short) are as follows:

Segmenting linearization: according to the principle of segmenting method, $t$ is divided into many small segments, when step length $\triangle t$ is small enough, because $A$ and $B$ changed very little within small segment $\left[t_{i}, t_{i}+\triangle t\right], A$ and $B$ can be regarded as fixed values, and takes $A$ and $B$ of $t=$ $t_{i}+0.5 \triangle t$ as the fixed values of $A$ and $B$ within $\left[t_{i}, t_{i}+\triangle t\right]$, namely (2) can be regarded as linear second-order ordinary differential equation within $\left[t_{i}, t_{i}+\triangle t\right]$.

Solving linear second-order ordinary differential equation: within $\left[t_{i}, t_{i}+\triangle t\right]$, based on Laplace transform, (2) is transformed to:

$$
Y(s)=\frac{y_{i} s^{2}+\left(y_{i} A+v_{i}\right) s+B}{s^{2}(s+A)}
$$

where $y_{i}$ is the initial value of $y$ within $\left[t_{i}, t_{i}+\triangle t\right], v_{i}$ is the initial value of $d y / d t$ within $\left[t_{i}, t_{i}+\triangle t\right]$, namely $(d y / d t)_{i}$.

The analytical solution of (3) within $\left[t_{i}, t_{i}+\triangle t\right]$ is obtained by means of inverse Laplace transform:

$$
\left\{\begin{array}{l}
\frac{d^{2} y}{d t^{2}}=\left(B-A v_{i}\right) e^{-A t} \\
\frac{d y}{d t}=\frac{B}{A}+\left(v_{i}-\frac{B}{A}\right) e^{-A t} \\
y=y_{i}+\frac{v_{i}}{A}+\frac{B}{A} t-\frac{B}{A^{2}}+\frac{1}{A}\left(\frac{B}{A}-v_{i}\right) e^{-A t}
\end{array}\right.
$$

Getting recursive formulas: the solution of $t=t_{i}+\Delta t$ is expressed by the solution of $t=t_{i}$, the following recursive formulas are got:

$$
\left\{\begin{array}{l}
\left(\frac{d^{2} y}{d t^{2}}\right)_{i+1}=\left[B-A\left(\frac{d y}{d t}\right)_{i}\right] e^{-A \Delta t} \\
\left(\frac{d y}{d t}\right)_{i+1}=\frac{B}{A}-\frac{1}{A}\left(\frac{d^{2} y}{d t^{2}}\right)_{i+1} \\
y_{i+1}=y_{i}+\frac{B}{A} \Delta t+\frac{1}{A}\left(\frac{d y}{d t}\right)_{i}-\frac{1}{A}\left(\frac{d y}{d t}\right)_{i+1}
\end{array}\right.
$$

Numerical algorithm: takes a suitable step length $\triangle t$, gets $A$ and $B$ of $t=t_{i}$ by initial values $y_{i}$ and $v_{i}$, and takes $A$ and $B$ of $t=t_{i}$ as the fixed values of $A$ and $B$ within $\left[t_{i}\right.$, $\left.t_{i}+0.5 \triangle t\right]$. Obtains the prediction solution of $t=t_{i}+0.5 \triangle t$ by substituting the fixed values of $A$ and $B$ within $\left[t_{i}, t_{i}+0.5\right.$ $\triangle t]$ and step length $0.5 \triangle t$ into recursive formulas. According to the prediction solution, gets $A$ and $B$ of $t=$ $t_{i}+0.5 \triangle t$, and takes $A$ and $B$ of $t=t_{i}+0.5 \triangle t$ as the fixed values of $A$ and $B$ within $\left[t_{i}, t_{i}+\triangle t\right]$. Obtains the numerical solution of $t=t_{i}+\triangle t$ by again substituting the fixed values of $A$ and $B$ within $\left[t_{i}, t_{i}+\triangle t\right]$ and step length $\triangle t$ into recursive formulas. Repeats recursive process, until obtains all numerical solution. In the beginning, takes $y_{0}$ and $v_{0}$ as initial values.

\section{COMPUTATION RESULTS AND VALIDATION}

In the following examples, numerical solutions are first obtained by SRM, and then numerical solutions are obtained based on fourth-order Runge-Kutta algorithm ODE45 improved by Felhberg in MATLAB (or RKF for short). For stiff equation, because RKF loses effectiveness, numerical solutions are obtained based on Gear multistep reverse numerical integration algorithm ODE15s in MATLAB (or Gear for short). RKF and Gear are adaptive variable step length algorithm, unless otherwise stated, the relative error of RKF and Gear are set to $10^{-6}$ in order to ensure computation accuracy. Finally, two kinds of numerical solutions are compared to verify the effectiveness of SRM.

\section{A. Van der Pol Oscillator Equation}

Van der Pol self-excitation oscillator equation is as follows:

$$
\left\{\begin{array}{l}
\frac{d^{2} y}{d t^{2}}+2\left(y^{2}-1\right) \frac{d y}{d t}+y=0 \\
y(0)=-0.2, \frac{d y}{d t}(0)=-0.7
\end{array}\right.
$$

Lets $A=2\left(y^{2}-1\right), B=-y$, takes $\triangle t=0.04$, the numerical solutions of SRM and RKF are shown in Fig .1. At this time, the RKF computation points are 1377, and the SRM computation points are only 751 , which are about half of the RKF computation points.

\section{B. Duffing Oscillator Equation}

Duffing forced oscillator equation is as follows:

$$
\frac{d^{2} y}{d t^{2}}+\frac{d y}{d t}-y+y^{3}=8 \cos 0.7 t, y(0)=1.0, \frac{d y}{d t}(0)=0.5
$$

Lets $A=1, B=8 \cos 0.7 t+y-y^{3}$, takes $\triangle t=0.06$, the numerical solutions of SRM and RKF are shown in Fig .2. At this time, the RKF computation points are 2101, and the SRM computation points are only 667, which are less than one-third of the RKF computation points.
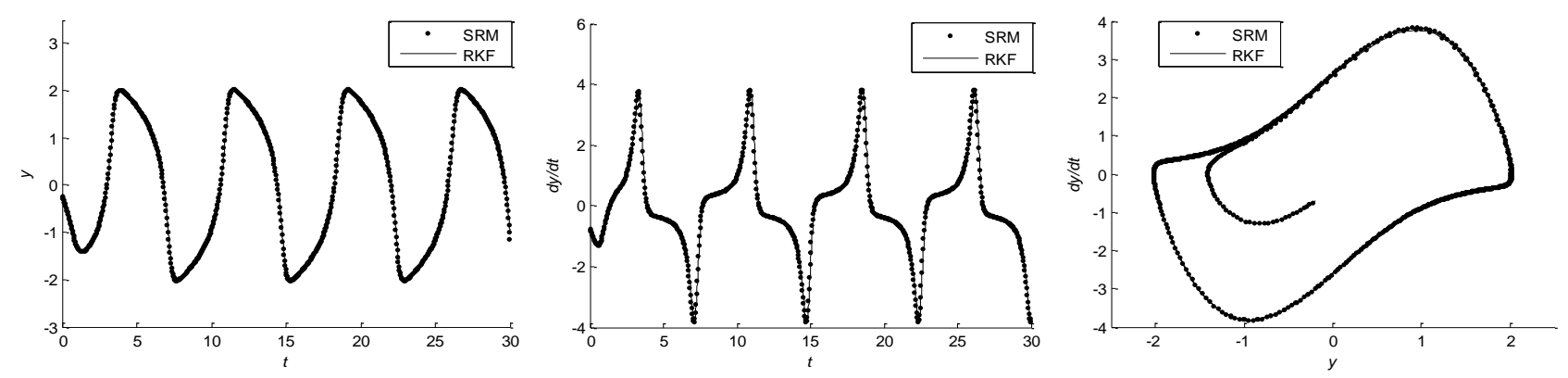

Figure 1. The SRM and RKF numerical solutions of Van der Pol self-excited oscillator equation when $\triangle t=0.04$. 


\section{Van der Pol-Duffing Oscillator Equation}

Van der Pol-Duffing forced oscillator equation is as follows:

$$
\frac{d^{2} y}{d t^{2}}+\left(y^{2}-1\right) \frac{d y}{d t}-y+y^{3}=\cos 0.7 t, y(0)=0.1, \frac{d y}{d t}(0)=-0.2
$$

Lets $A=y^{2}-1, B=\cos 0.7 t+y-y^{3}$, takes $\triangle t=0.09$, the numerical solutions of SRM and RKF are shown in Fig .3. At this time, the RKF computation points are 1649, and the SRM computation points are only 556, which are about one-third of the RKF computation points.

Fig .1-Fig .3 show: when step length $\triangle t=0.04-0.09$, the numerical solutions obtained by SRM and RKF have been very close to, almost exactly the same, but the calculating points are greatly reduced, indicates that SRM is very effective to solve Van der Pol-Duffing oscillator equation, not only method is simple, programming is easy, but result is accurate. If step length $\triangle t$ is further reduced, the numerical solutions obtained by SRM will be more accurate. Meanwhile, their phase diagrams show that (6)(8) all have stable limit cycle, indicates that their movement all finally are periodic vibration.

\section{STEP LENGTH SELECTION}

\section{A. Conventional Computation Step Length}

In numerical computation, the absolute error of numerical solution is jointly decided by truncation error and rounding error. According to the principle and methodof SRM, the reason caused truncation error by SRM is that $A$ and $B$ are linearized to fixed values within small segment $\left[t_{i}, t_{i}+\triangle t\right]$, and the recursive formulas are accurate. Therefore, the smaller step length $\triangle t$ is, the smaller truncation error is, the higher computation accuracy is, but recursive step number will increase correspondingly, thus computation workload will increase, and rounding error will also increase slightly. So a suitable step length should be selected according to accuracy requirement in computation.

For (6), takes $\triangle t=0.1$, the numerical solutions of SRM and RKF are shown in Fig .4. At this time, the RKF computation points are 1377, and the SRM computation points are only 300 , which are less than a quarter of the RKF computation points, because the SRM computation points are too little, the numerical solutions obtained have small error, and error gradually increases as $t$ increases.
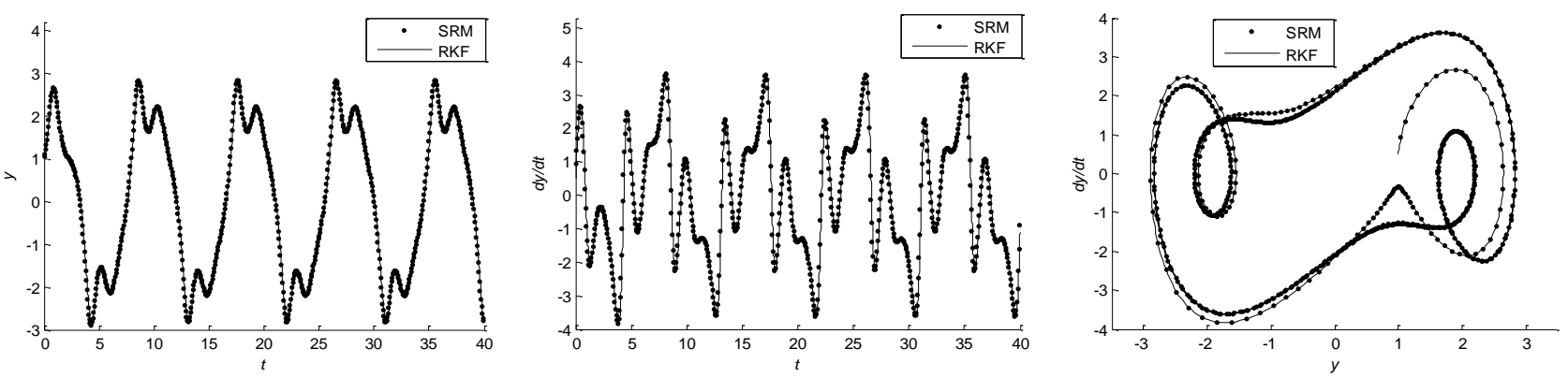

Figure 2. The SRM and RKF numerical solutions of Duffing forced oscillator equation when $\triangle t=0.06$.
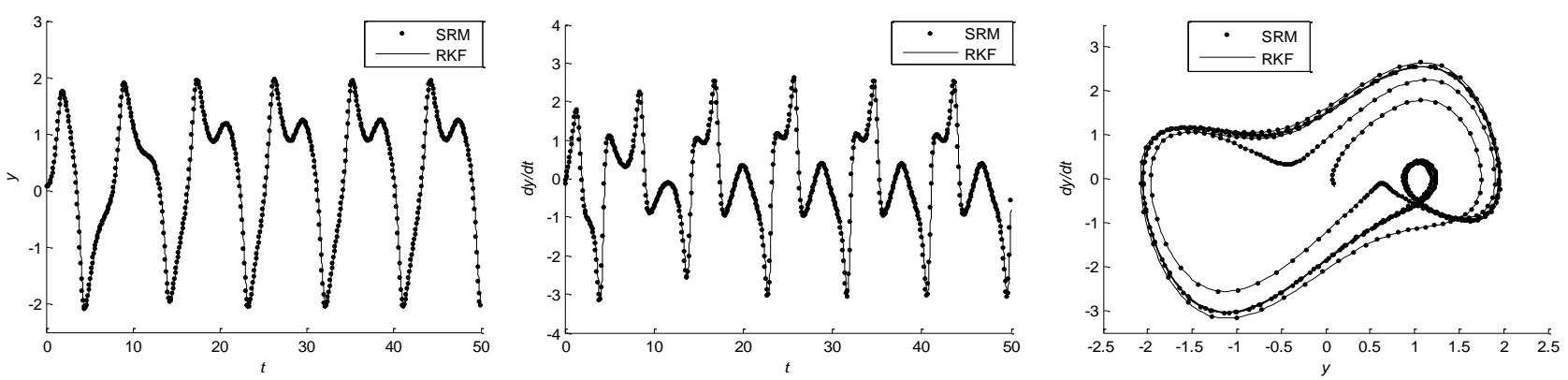

Figure 3. The SRM and RKF numerical solutions of Van der Pol-Duffing forced oscillator equation when $\triangle t=0.09$
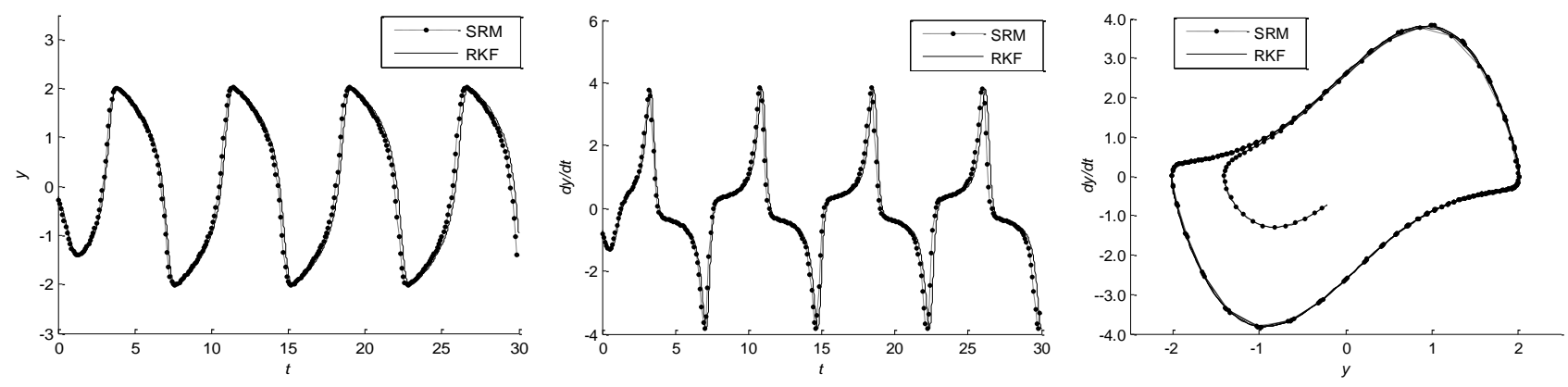

Figure 4. The SRM and RKF numerical solutions of Van der Pol self-excited oscillator equation when $\triangle t=0.1$. 
Fig .4 shows: when step length $\triangle t$ is too large, because computation points are too little, causes larger linearization truncation error. In most cases, Van der Pol-Duffing oscillator equation is a strong nonlinear equation, namely the nonlinearity of $A$ or $B$ in (2) is strong, so $\triangle t$ should not be taken too large in order to ensure computation accuracy. In conventional computation, taking step length $\triangle t=0.01$ - 0.05 can meet accuracy requirement, the stronger nonlinearity of $A$ or $B$ is, the smaller $\triangle t$ is taken. When the nonlinearity of $A$ and $B$ are weak, linearization error is very little, at this time, even if taking a larger step length $\triangle t$, computation accuracy can also meet requirement.

\section{B. Chaotic Motion Step Length}

Van der Pol-Duffing forced oscillator equation is as follows:

$$
\left\{\begin{array}{l}
\frac{d^{2} y}{d t^{2}}+0.2\left(y^{2}-1\right) \frac{d y}{d t}-y+y^{3}=0.53 \cos t \\
y(0)=0.1, \quad \frac{d y}{d t}(0)=-0.2
\end{array}\right.
$$

Lets $A=0.2\left(y^{2}-1\right), B=0.53 \cos t+y-y^{3}$, when relative error is default value $10^{-3}$, the numerical solutions of (13) are obtained by Gear and RKF. Takes $\triangle t=0.03$ and $\triangle t=0.01$, the numerical solutions are obtained by SRM. Takes relative error $10^{-6}$, the numerical solutions are obtained by RKF. These results are compared in Fig .5. Fig .5 shows: when relative error is $10^{-3}$, the numerical solutions of Gear and RKF have large error after $t>10$, especially when $t=25-35$, error is larger, but when relative error is $10^{-6}$, the numerical solutions of RKF are very accurate. Meanwhile, takes $\triangle t=0.03$, the numerical solutions of SRM have also large error after $t>30$, but takes $\triangle t=0.01$, the numerical solutions of SRM are also very accurate, almost coincide completely with the numerical solutions of RKF when relative error is $10^{-6}$. Fig .5 shows that (13) is in chaotic motion, the reaction to truncation error is very sensitive.

When there is chaotic motion, the size of truncation error may have a significant effect to chaotic motion, larger error may change the nature of solution, thus chaotic characteristics may be lost. Therefore, when solving the chaotic motion numerical solutions, step length $\triangle t$ should be taken a smaller value to reduce truncation error and improve computation accuracy, when taking $\triangle t=0.005-$ 0.01 , the results are generally desirable.

\section{Adaptive Variable Step Length}

When Van der Pol-Duffing oscillator equation is solved by SRM, fixed step length generally can meet computation requirement, adaptive variable step length method also can be used to further improve computation efficiency and reduce computation time.

The relative error is defined as follows:

$$
\Delta=\left|\frac{y_{i+1}^{(\Delta t / 2)}-y_{i+1}^{(\Delta t)}}{y_{i+1}^{(\Delta t / 2)}}\right|
$$

where $y_{i+1}^{(\Delta t / 2)}$ is the solution after computing two steps by means of half step length $0.5 \triangle t, y_{i+1}^{(\Delta t)}$ is the solution after computing one step by means of step length $\triangle t$.

Given a relative error $\varepsilon$, when $\triangle>\varepsilon$, the numerical solution is computed by means of half step length, until meeting $\triangle<\varepsilon$, takes final $y_{i+1}^{(\Delta t / 2)}$ as result. When $\triangle \leqslant \varepsilon$, the numerical solution is computed by means of double step length, until meeting $\triangle \geqslant \varepsilon$, then the numerical solution is computed once by means of half step length, takes final $y_{i+1}^{(\Delta t / 2)}$ as result.
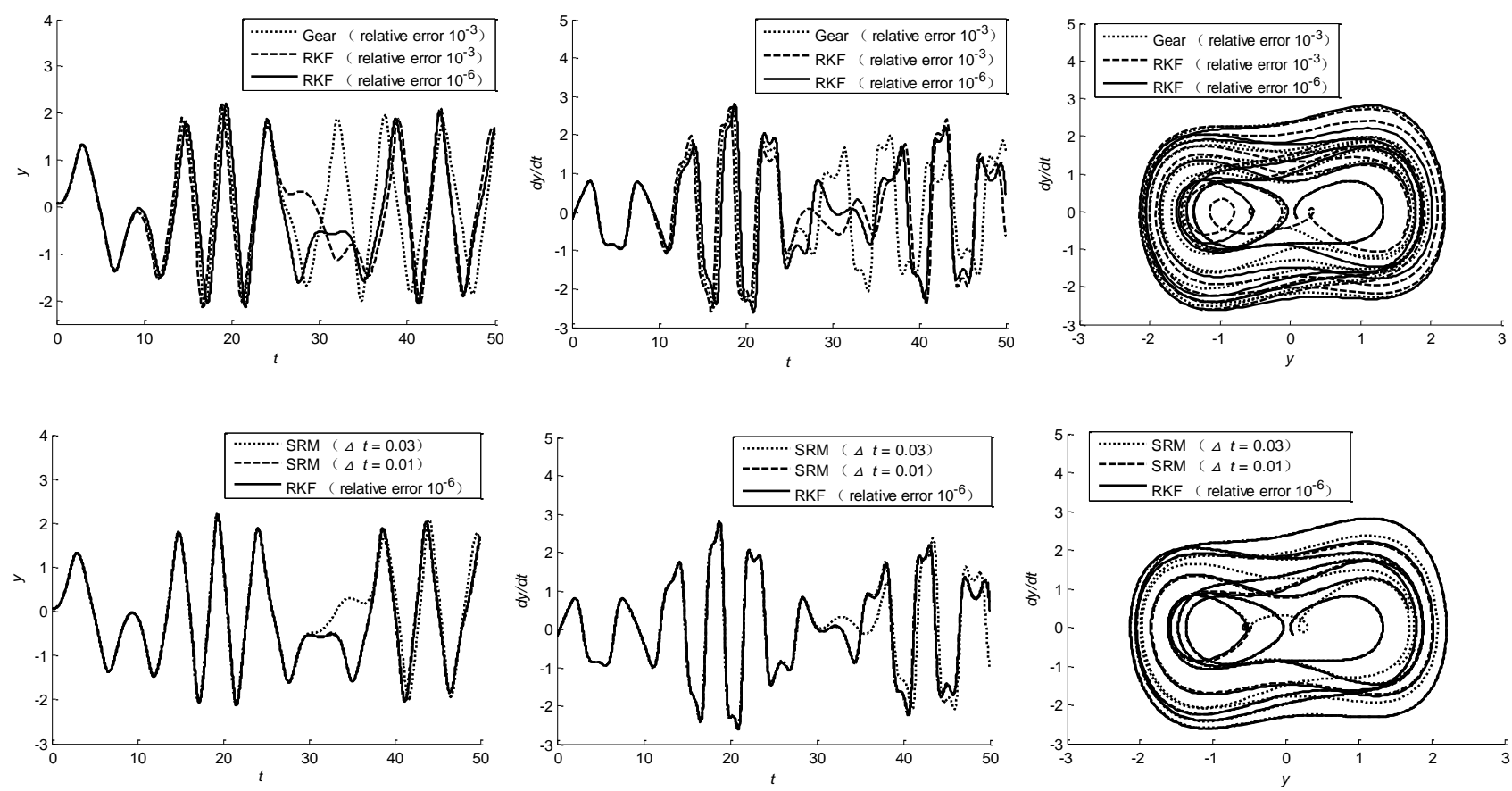

Figure 5. The SRM and RKF numerical solutions of Van der Pol forced oscillator in chaotic motion. 
Van der Pol self-excitation oscillator equation is as follows:

$$
\left\{\begin{array}{l}
\frac{d^{2} y}{d t^{2}}+1000\left(y^{2}-1\right) \frac{d y}{d t}+y=0 \\
y(0)=-0.2, \quad \frac{d y}{d t}(0)=-0.7
\end{array}\right.
$$

Lets $A=1000\left(y^{2}-1\right), B=-y$, based on adaptive variable step length method, when simulation time is 3000 and $\varepsilon=10^{-6}$, the numerical solutions of SRM and Gear are shown in Fig .6. At this time, the Gear computation points are 2036, and the SRM computation points are 2225, which are slightly more than the Gear computation points, the numerical solutions obtained by two methods are also very close to, almost exactly the same.

For adaptive variable step length method, when numerical solutions change quickly, automatically take smaller step length, and when numerical solutions change slowly, automatically take larger step length, not only ensure computation accuracy, but also improve computation efficiency and reduce computation time, of course, programming difficulty will slightly increase. This method is specially suitable for stiff equation or long simulation time.
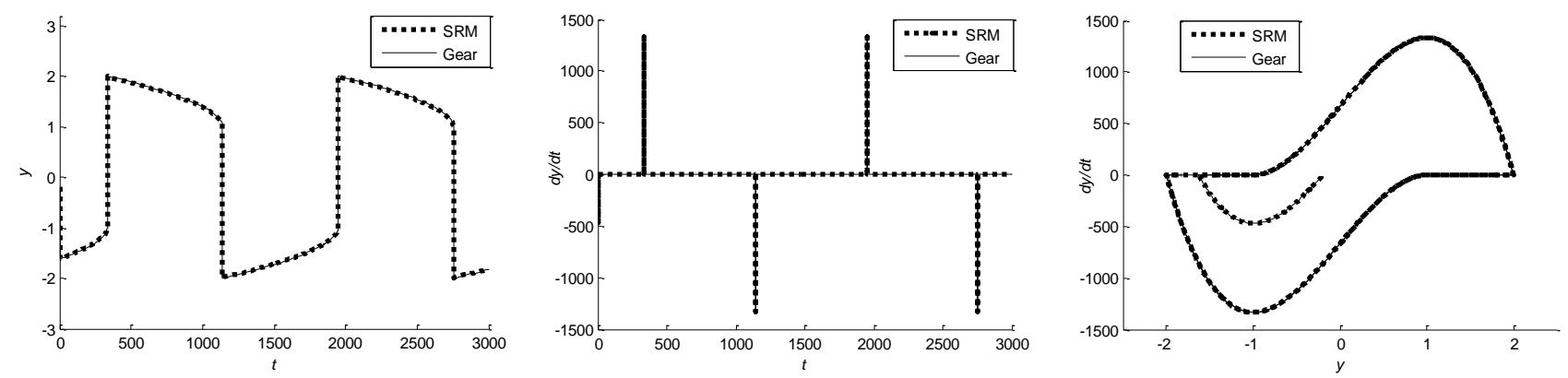

Figure 6. The SRM and Gear numerical solutions of Van der Pol stiff equation with long simulation time.

\section{CONCLUSIONS}

Segmenting recursion method is very effective to solve Van der Pol-Duffing oscillator equation, not only method is simple, programming is easy, but result is accurate, this method is suitable for strong nonlinear, weak nonlinear and stiff equation, it is a universal new numerical method to solve similar equation. In conventional computation, taking step length $0.01-0.05$ generally can obain more accurate results. In chaotic motion computation, in order to avoid losing chaotic characteristics, taking step length can $0.005-0.01$ generally can obain desirable results. For stiff equation or long simulation time, adaptive variable step length method can be used to improve computation efficiency.

\section{ACKNOWLEDGMENT}

This work was supported by the National Natural Science Foundation of China (Grant No.51179079)

\section{REFERENCES}

[1] B. Van der Pol and J. Van der Mark "The heartbeat considered as a relaxation oscillation, and an electrical model of the heart". Pholophical Magazine, 1928, vol. 6(38), pp. 763-775.

[2] V.V.Udilov, "Analysis of dynamics of nonlinear Duffing-van der Pol oscillator using versal models". Journal of Automation and Information Science, 2001, vol. 32(5), pp. 9-19.

[3] D. Storti and G. Per, "Phase-locked mode stability for coupled van der Pol oscillators". Journal of Vibration and Acoutics, 2001, vol. 122(3):,pp. 318-323.

[4] Y. Tsuda, H. Tamara, A. Sueoka, et al. "Chaotic behaviour of a nonlinear vibrating system with a retarded argument". JSME International Journal : Seris III, 1992, vol. 35(2), pp. 259-267.

[5] K. Moukam, S. Bowong and C. Tchawoua, et a1. "Stranhe attractors and chaos control in a Duffing-VanderPol oscillator with two eternal periodic forces". Journal of Sound and Vibration, 2004, vol. 277(4), pp. 783-799.

[6] A. M. Wazwaz, "A new method for solving singular initial value problems in the second-order ordinary differential equations", Appl. Math. Comput. 2002, vol. 128, pp. 45-57.

[7] Q. Y. Li and Y. X. Ren, "S-polar sets of super-Brownian motions and solutions of nonlinear differential equations", Science in China Ser. A: Mathematics, 2005, vol. 48, pp. 1683-1695.

[8] C. M. Bender, K. A. Milton and S. S. Pinsky, et al., "A new perturbative approach to nonlinear problems", J. Math. Phys. 1989, vol. 30: pp. 1447-1455.

[9] J. I. Ramos, "Linearized methods for ordinary differential equations", Appl. Math. Comput. 1999, vol. 104, pp. 109-129.

[10] S. C. Wafo and F. M. Mahomed, "Linearization criteria for a system of second-order ordinary differential equations", International Journal of Non-Linear Mechanics. 2001, vol. 36, pp. 671-677.

[11] H. D. Cruz, R. J. Biscay and F. Carbonell, et al., "A higher order local linearization method for solving ordinary differential equations", Applied Mathematics and Computation. 2007, vol. 185, pp. 197-212.

[12] S. V. Meleshko, "On linearization of third-order ordinary differential equations”, J. Phys. A. 2006, vol. 39, pp. 15135-15145.

[13] J. F. Cao, "Nonlinear system theory and application", Xi'an Jiaotong University Press, Xi'an, 2001.

[14] H. L. Wang, and Q. C. Zhang, "Nonlinear dynamics theory and its application”. Tianjin Science and Technology Press, Tianjin, 2002.

[15] J. H. He, "Approximate nonlinear analysis methods in engineering and scientific computation". Henan Science and Technology Press, Zhengzhou, 2002.

[16] S. Y. Zhang and Z. C. Deng, "The geometric integration theory and application of nonlinear dynamics system". Northwestern Polytechnical University Press, Xi'an, 2005.

[17] S. Q. Zhu, "Approximate analysis of nonlinear systems". National Defense Industry Press. Beijing, 1980.

[18] D. Y. Xie, “Asymptotic method”, China Friendship Press, Beijing, 1983. 